\title{
Rating Locomotive Crew Diesel Emission Exposure Profiles Using Statistics and Bayesian Decision Analysis
}

\author{
Supplemental Materials
}

\section{Additional Text}

\section{Selection of OELS}

Australian Institute of Occupational Hygienists $(\mathrm{AIOH}, 2013)$ guideline for diesel particulate matter (to be measured as EC) was designed to control the irritant effects associated with exposure to diesel emissions. The AIOH noted that "such a level should result in a reduced lung cancer outcome if such a carcinogenic effect is attributable to DPM exposure".

The California guideline of $0.02 \mathrm{mg} / \mathrm{m}^{3}$ was used for evaluating EC occupational exposures (CDHS, 2002). The U.S. Environmental Protection Agency (EPA) established a "Reference Concentration" (RfC) for "diesel exhaust" of $0.005 \mathrm{mg} / \mathrm{m}^{3}$, which was intended as a guideline for the "continuous inhalation exposure to the human population, including sensitive subgroups, ... [that is] ... likely to be without appreciable risks of deleterious noncancer effects during a lifetime" (EPA, 2002). The EPA RfC can provide additional insight into the level of risk experienced by the CSXT locomotive crews. (The RfC was expressed in terms of DPM, but the EPA felt that although not all EC originates with diesel engines, EC is an adequate surrogate for DPM (EPA, 2002; pages 2-100 to 2-101).)

The Mine Safety and Health Administration (MSHA) adopted a PEL for diesel emissions of 0.16 $\mathrm{mg} / \mathrm{m}^{3}$, to be measured as total carbon (MSHA, 2013). This PEL was primarily intended for use in dieselized underground mines. To minimize the contribution of non-diesel emission-related sources of carbon (e.g., the dust in an underground coal mine), MSHA specified including a "sub-micron impactor" in the sampling train to eliminate the generally larger particles generated when mining. This type of particle pre-separator was not used in the CSXT studies when sampling for total carbon. Consequently, it is possible that the total carbon measurements in this dataset were slightly overestimated when comparing to the MSHA PEL.

\section{Methods}

\section{Assigning an AIHA Exposure Rating}

The AIHA exposure category assignment can be determined by considering the confidence interval for the sample estimate and/or by evaluating the BDA category decision probabilities. For BDA, the following rule-of-thumb was used: low certainty whenever the highest decision probability was $<0.5$, medium certainty when it was $>0.5$ but $<0.75$, and high certainty when it is $>0.75$. These are not bright lines regarding the certainty level, but merely guidelines. If the sample 95th percentile is nearly equal to the dividing line between two adjacent exposure categories the decision probabilities for the two categories will be similar. In this instance, it is permissible to assign both categories and to sum the decision probabilities for assigning confidence. For example, "category $0 / 1$, high certainty" would indicate that the true 95th percentile could be either in the range for category 0 or 1 , and the combined decision probability exceeds 0.75 .

Goodness-of-fit Evaluation.

The null or default hypothesis is that the distribution is lognormal. The null hypothesis is rejected if there is compelling evidence that it is incorrect and the alternative hypothesis is most likely correct. Departures from the best-fit line - for example, unexpected curves in the plotted data or clusters of data suggest that the unimodal lognormal model may not be a good fit, and that the dataset may have come from two or more different populations of data. A professional judgment call is often necessary if the data pass a subjective evaluation, but fail an objective test. 


\section{RESULTS}

\section{Analysis using BDA}

When using BDA the primary assumption is that the underlying exposure profile can be adequately modeled by the lognormal distribution. If the true 95th percentile was known the decision probability for one category would be 1.00 and zero for the remaining categories. However, for a typical dataset the decision probabilities are generally much less than 1.00 , reflecting both the sample size and the variability in the data. In instances where the data are very low or very high, relative to the OEL, the decision probability in the lowest or highest category may round to 1.00 , but is not truly unity.

\section{Determinants of Exposure}

Effect of Passing through Tunnels. The number of tunnels passed through during the run was recorded for many of the EC measurements. The majority of the records did not have an entry for this variable; most likely for most of these no tunnel was encountered. In Figure 4, the EC levels were plotted versus the number of tunnels. There was no discernible increasing trend in the EC levels with an increasing number of tunnels encountered during the run.

Effect of Locomotive Manufacturer, Class, and Model. There was considerable overlap of the EC data when plotted by manufacturer, locomotive class (4-axle versus 6-axle), and locomotive model (see Figures 5-10). The figures show that most of the EC and TC measurements, regardless of locomotive manufacturer or class, were below the California guideline of $0.02 \mathrm{mg} / \mathrm{m}^{3}$ for elemental carbon and the MSHA (mining) PEL of $0.16 \mathrm{mg} / \mathrm{m}^{3}$, respectively. There may be differences in EC levels related to manufacturer, class, and model. However, there was no further analysis of these factors given the generally low exposures and the fact that a sizable fraction of the locomotive crew EC exposures originates from ambient sources outside of the locomotive.

\section{ANOVA}

Table VIII contains the results of an Analysis of Variance (ANOVA) of the combined effects of locomotive position (lead versus trailing) and window status (open versus closed) on the EC levels. 


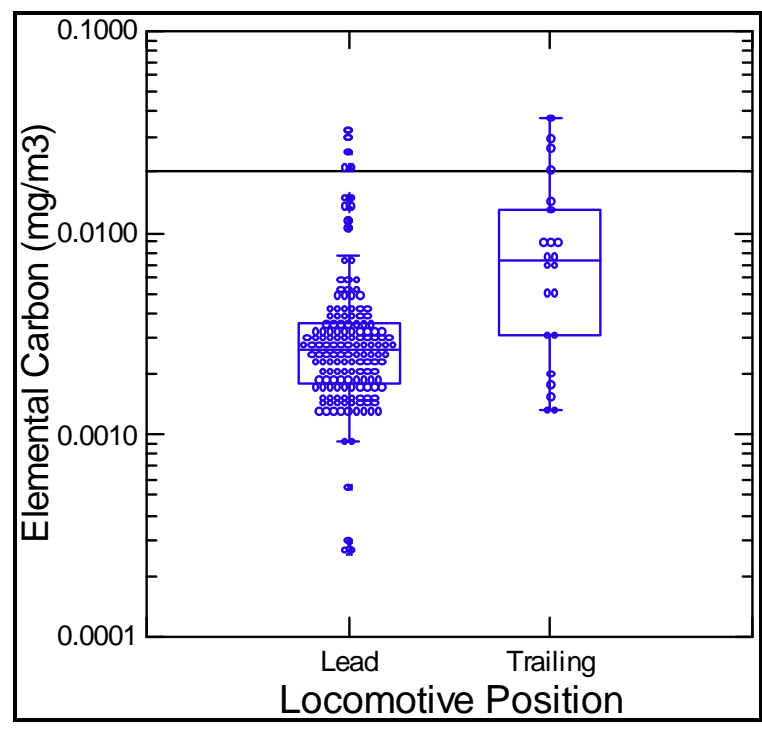

Figure 1: Box-whisker plot of the elemental carbon data, by the position of the sampled locomotive. (The horizontal line indicates the California guideline of $0.02 \mathrm{mg} / \mathrm{m}^{3}$.)

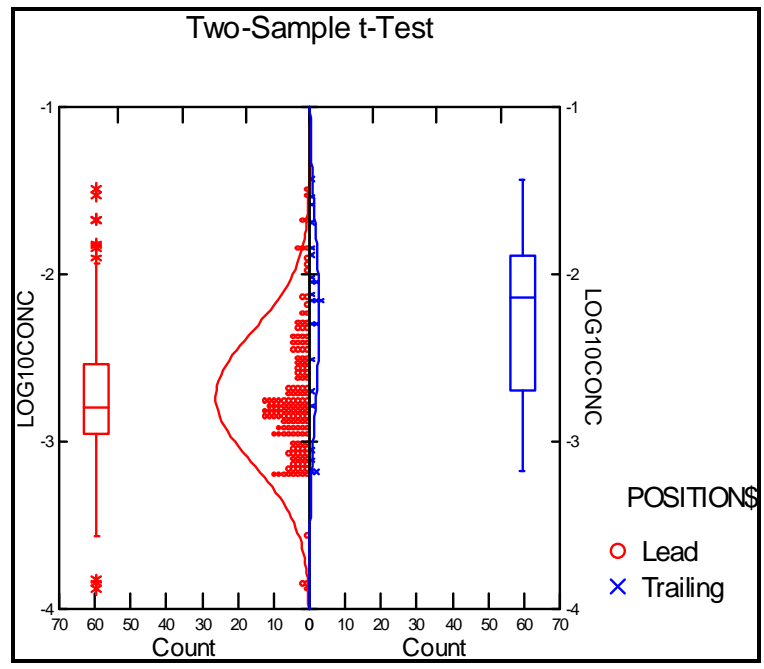

Figure 3: Two-sample, t-test comparison of the $\log _{10}$-transformed levels for EC levels in the lead versus trailing locomotive. (Non-detects were replaced with half of the detection limit.) The distributions were significantly different $(p<0.001)$ with the CSXT distribution being slight lower. (The $\mathrm{p}$-value was also less than 0.001 without replacement of the non-detects.)

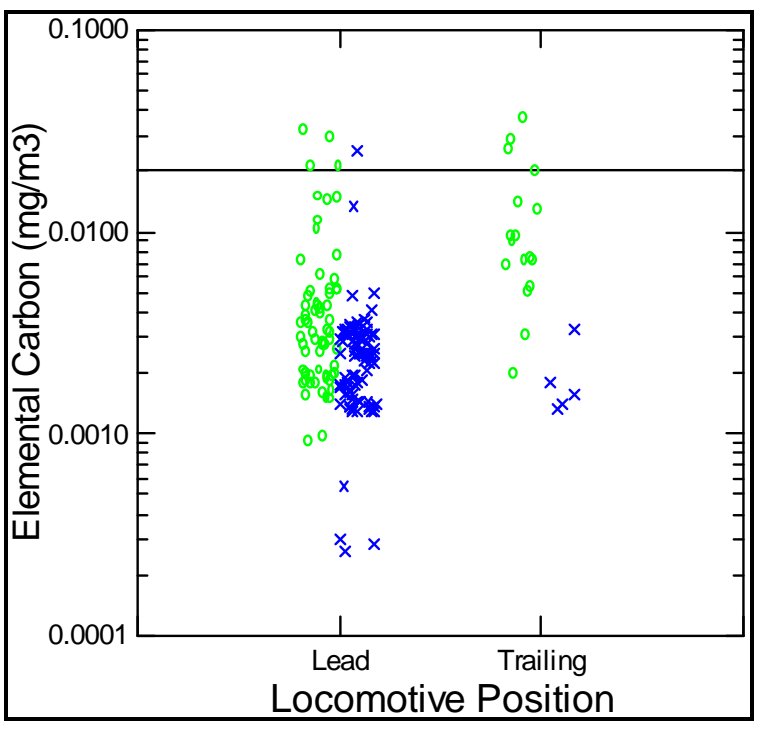

Figure 2: Jittered-dot density plot of the elemental carbon data, by the position of the sampled locomotive. (The horizontal line indicates the California guideline of $0.02 \mathrm{mg} / \mathrm{m}^{3}$.) $\quad(0=$ detect; $x=$ non-detect)

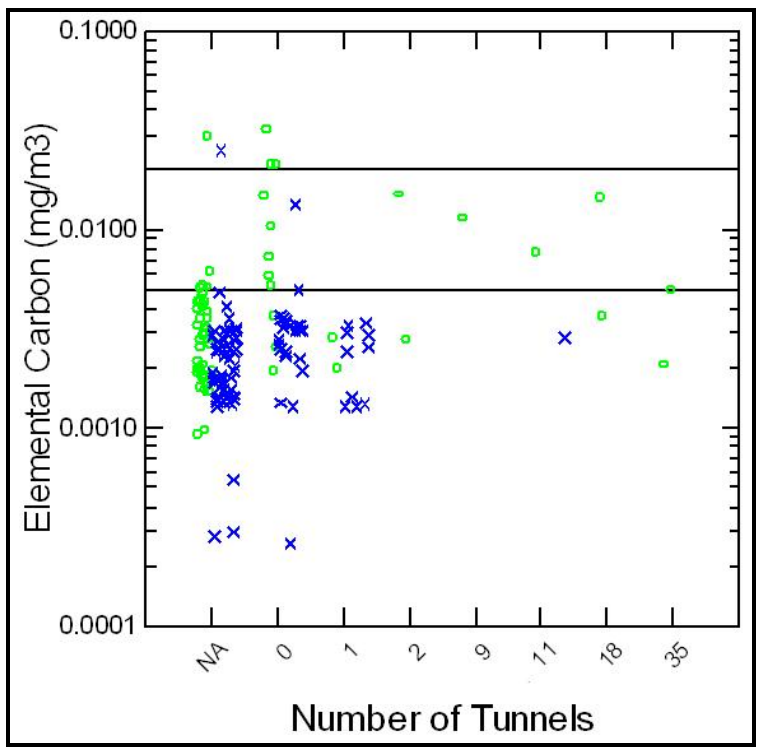

Figure 4: Jittered dot-density plot of the elemental carbon data, by the number of tunnels encountered during the run. (The horizontal lines indicates the California guideline of $0.02 \mathrm{mg} / \mathrm{m}^{3}$ and the EPA Reference Concentration of $\left.0.005 \mathrm{mg} / \mathrm{m}^{3}\right)(\mathrm{n}=156)$ ( $\mathrm{o}=$ detect; $\mathrm{x}=$ non-detect) 


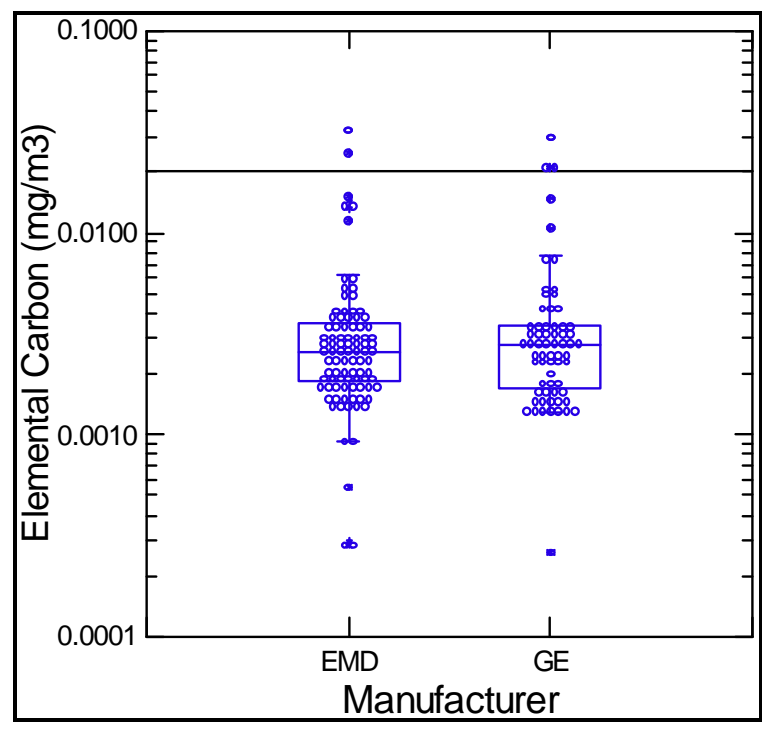

Figure 5: Box-whisker plot of the elemental carbon data for the lead locomotive, by manufacturer. (The horizontal line indicates the California guideline of $0.02 \mathrm{mg} / \mathrm{m}^{3}$.) $(\mathrm{n}=156)$

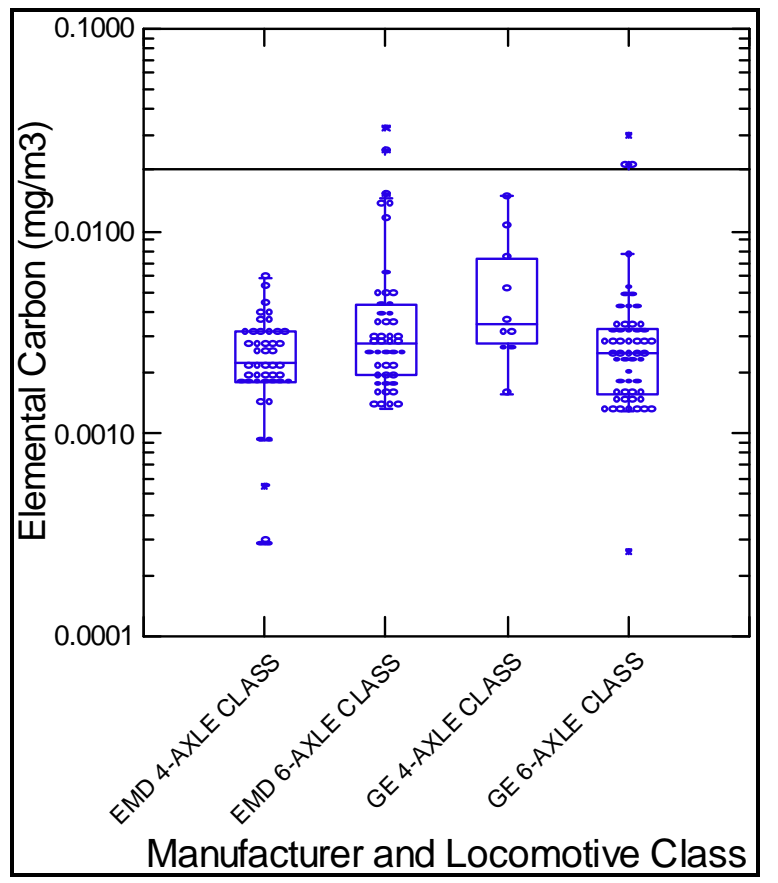

Figure 7: Box-whisker plot of the elemental carbon data for the lead locomotive, by manufacturer and locomotive class. (The horizontal line indicates the California guideline of $0.02 \mathrm{mg} / \mathrm{m}^{3}$.) $(\mathrm{n}=156)$

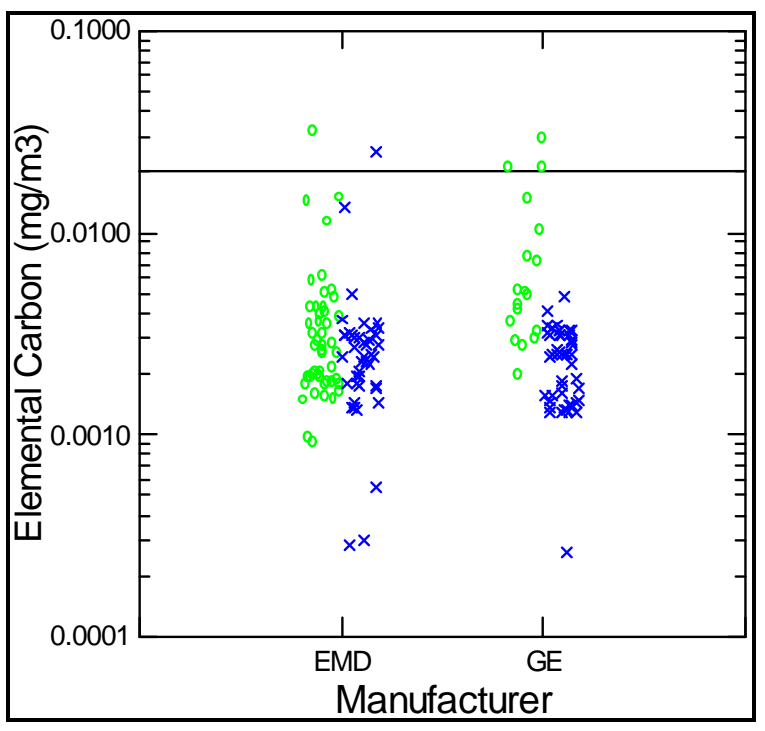

Figure 6: Jittered dot-density plot of the elemental carbon data for the lead locomotive, by manufacturer. ( $0=$ detect; $x=$ non-detect) (The horizontal line indicates the California guideline of $0.02 \mathrm{mg} / \mathrm{m}^{3}$.) $(n=156)$

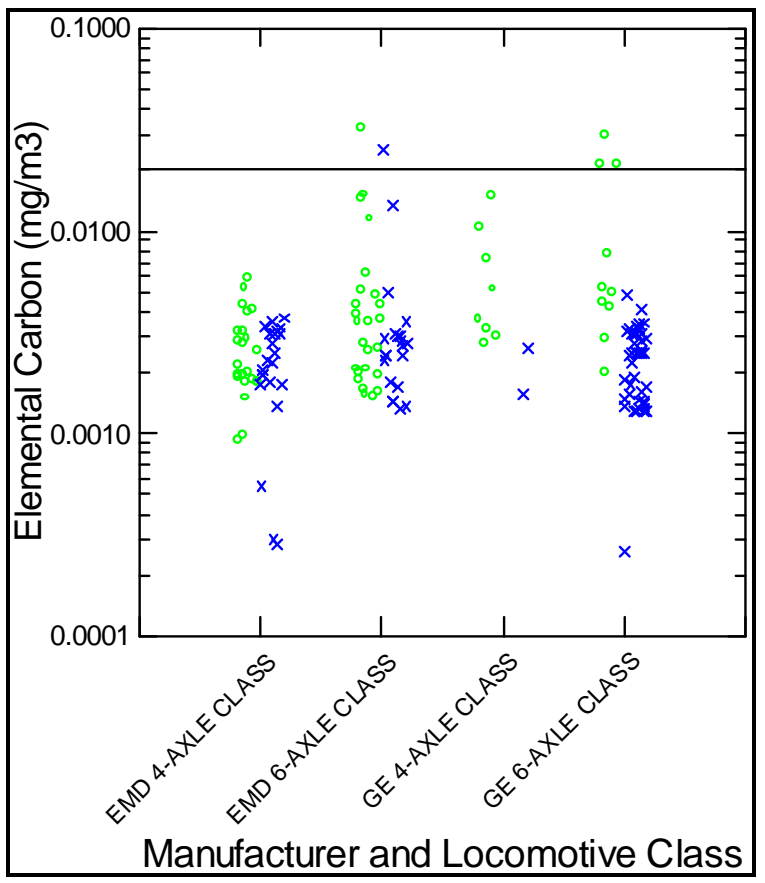

Figure 8: Jittered dot-density plot of the elemental carbon data for the lead locomotive, by manufacturer and locomotive class. ( $0=$ detect; $x=$ non-detect) (The horizontal line indicates the California guideline of $0.02 \mathrm{mg} / \mathrm{m}^{3}$.) $(\mathrm{n}=156)$ 


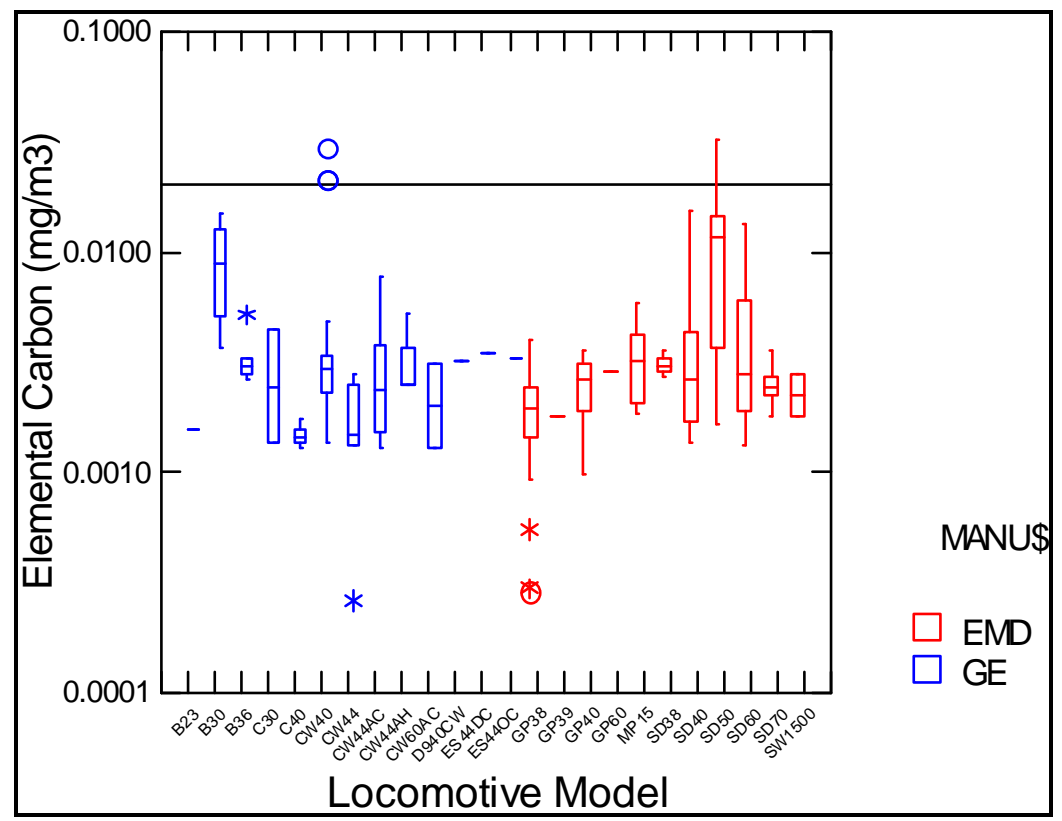

Figure 9: Box-whisker plot of the elemental carbon data for the lead locomotive, by locomotive model. (The horizontal line indicates the California guideline of $0.02 \mathrm{mg} / \mathrm{m}^{3}$.)

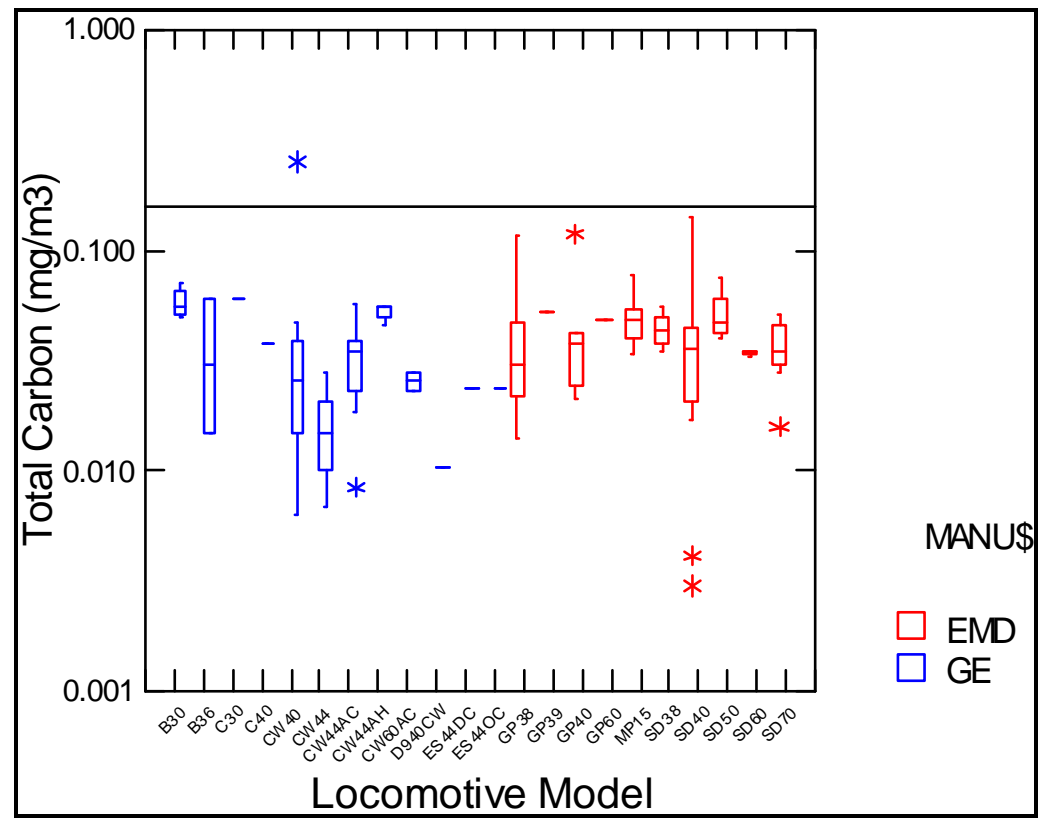

Figure 10: Box-whisker plot of the total carbon data for the lead locomotive, by locomotive model. (The horizontal line indicates the MSHA PEL for mining of $0.16 \mathrm{mg} / \mathrm{m}^{3}$.) 


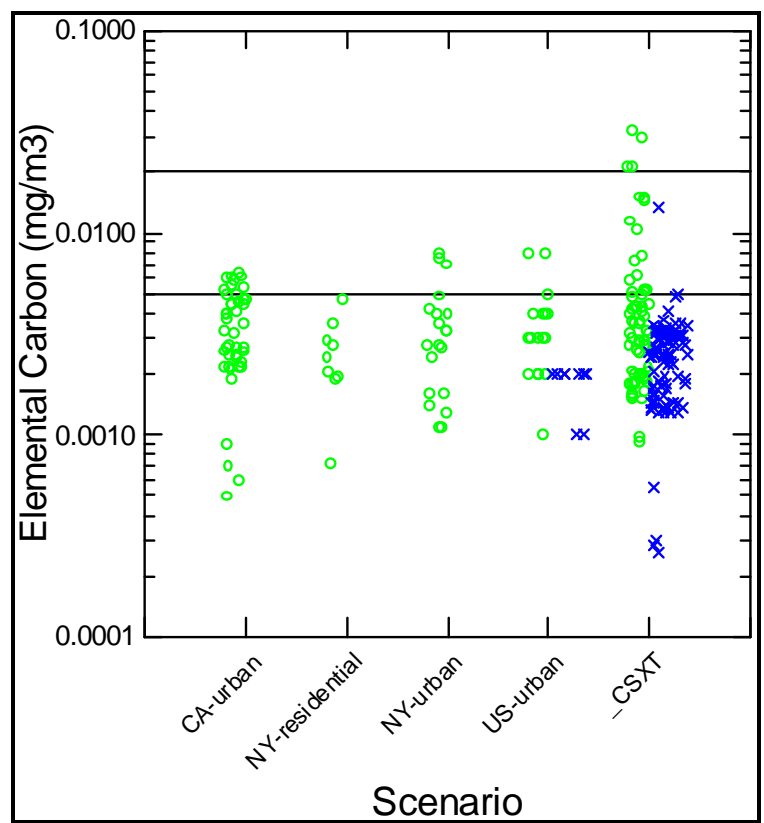

Figure 11: Jittered dot-density plot of the elemental carbon data for CSXT lead locomotives vs data from Los Angeles, New York, and a collection of U.S. cities. The horizontal lines represent the California guideline of $0.02 \mathrm{mg} / \mathrm{m}^{3}$ and the EPA Reference Concentration of $0.005 \mathrm{mg} / \mathrm{m}^{3}$. (o=detect; $\mathrm{x}=$ non-detect) 
Table I: Occupational exposure limits (OEL). The underlined OELs represent the CSXT working OELs. Unless otherwise indicated with a " $\mathrm{C}$ " for ceiling limit, all OELs are for an 8-hour full-shift.

\begin{tabular}{|c|c|c|c|}
\hline Substance & OEL & Source & Notes \\
\hline \multicolumn{4}{|c|}{ Diesel Particulate Emissions } \\
\hline \multirow{3}{*}{ elemental carbon (EC) } & $0.005 \mathrm{mg} / \mathrm{m}^{2}$ & EPA (2002) & $\begin{array}{l}\text { The EPA Reference Concentration (RfC) is a } \\
\text { public exposure limit; upper limit for the } \\
\text { average exposure. }\end{array}$ \\
\hline & $0.02 \mathrm{mg} / \mathrm{m}^{3}$ & CDHS (2002) & $\begin{array}{l}\text { California guidelines; occupational exposure } \\
\text { limit; upper limit for full-shift exposures. }\end{array}$ \\
\hline & $0.1 \mathrm{mg} / \mathrm{m}^{3}$ & AIOH (2013) & $\begin{array}{l}\text { occupational exposure limit; upper limit for } \\
\text { full-shift exposures. }\end{array}$ \\
\hline total carbon $(\mathrm{TC})$ & $0.16 \mathrm{mg} / \mathrm{m}^{3}$ & MSHA (2013) & \\
\hline \multicolumn{4}{|c|}{ Aromatics (BTEX) } \\
\hline benzene & $\begin{array}{l}1 \mathrm{ppm} \\
0.1 \mathrm{ppm} \\
0.5 \mathrm{ppm} \\
\end{array}$ & $\begin{array}{l}\text { OSHA (2012) } \\
\text { NIOSH (2004) } \\
\text { ACGIH (2012) }\end{array}$ & 1 \\
\hline toluene & $\begin{array}{r}100 \mathrm{ppm} \\
100 \mathrm{ppm} \\
20 \mathrm{ppm} \\
\end{array}$ & $\begin{array}{l}\text { OSHA (2012) } \\
\text { NIOSH (2004) } \\
\text { ACGIH (2012) }\end{array}$ & \\
\hline ethyl benzene & $\begin{array}{r}100 \mathrm{ppm} \\
100 \mathrm{ppm} \\
20 \mathrm{ppm} \\
\end{array}$ & $\begin{array}{l}\text { OSHA (2012) } \\
\text { NIOSH (2004) } \\
\text { ACGIH (2012) }\end{array}$ & \\
\hline xylene & $\begin{array}{l}100 \mathrm{ppm} \\
100 \mathrm{ppm} \\
100 \mathrm{ppm} \\
\end{array}$ & $\begin{array}{l}\text { OSHA (2012) } \\
\text { NIOSH (2004) } \\
\text { ACGIH (2012) }\end{array}$ & \\
\hline \multicolumn{4}{|c|}{ Aldehydes } \\
\hline acetaldehyde & $\frac{200 \mathrm{ppm}}{25 \mathrm{ppm} \mathrm{C}}$ & $\begin{array}{l}\text { OSHA (2012) } \\
\text { ACGIH (2012) }\end{array}$ & \\
\hline benzaldehyde & $\underline{2 \mathrm{ppm}}$ & AIHA (2011) & \\
\hline formaldehyde & $\begin{array}{c}\underline{0.75 \mathrm{ppm}}, 2 \mathrm{ppm} \mathrm{C} \\
0.016 \mathrm{ppm} \\
0.3 \mathrm{C}\end{array}$ & $\begin{array}{l}\text { OSHA (2012) } \\
\text { NIOSH (2004) } \\
\text { ACGIH (2012) }\end{array}$ & 1 \\
\hline glutaraldehyde & $\frac{0.05 \mathrm{ppm} \mathrm{C}}{0.2 \mathrm{ppm} \mathrm{C}}$ & $\begin{array}{l}\text { ACGIH (2012) } \\
\text { NIOSH (2004) }\end{array}$ & $\begin{array}{l}2 \\
1\end{array}$ \\
\hline \multicolumn{4}{|c|}{ Gases } \\
\hline carbon monoxide & $\begin{array}{l}50 \mathrm{ppm} \\
35 \mathrm{ppm} \\
25 \mathrm{ppm} \\
\end{array}$ & $\begin{array}{l}\text { OSHA (2012) } \\
\text { NIOSH (2004) } \\
\text { ACGIH (2012) }\end{array}$ & \\
\hline nitrogen dioxide & $\begin{array}{l}5 \text { ppm C } \\
1 \text { ppm STEL } \\
\underline{0.2 \mathrm{ppm}}\end{array}$ & $\begin{array}{l}\text { OSHA (2012) } \\
\text { NIOSH (2004) } \\
\text { ACGIH (2012) }\end{array}$ & 3 \\
\hline
\end{tabular}

1 - The NIOSH REL was devised under NIOSH's "old policy" for known or suspected carcinogens where the REL was generally set at the "lowest feasible concentration" (NIOSH, 2004). A revised REL devised under the new policy has not be issued.

2 - There is no OSHA PEL for glutaraldehyde. The TLV was used for comparison even though it is a ceiling limit and the measurements are partial-shift to full-shift.

3 - In 2012 the ACGIH reduced the TLV from 3 ppm to $0.2 \mathrm{ppm}$. The basis for the new TLV is irritation of the lower respiratory tract. 
Table II: Number of measurements collected each year.

\begin{tabular}{|c|c|c|c|c|c|c|c|c|c|c|c|c|c|c|c|c|c|c|c|c|c|c|c|}
\hline Class & Substance & 웍 & ने & నू & ભ̆ & $\begin{array}{l}\text { ஓे } \\
\text { ने }\end{array}$ & คू & $\begin{array}{l}\text { gे } \\
\text { बे }\end{array}$ & ڤ్ & $\begin{array}{l}\infty \\
\stackrel{2}{\Omega} \\
\stackrel{-}{-1}\end{array}$ & ஓे & 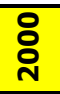 & ㅎㅇㅇ & $\begin{array}{l}\text { N } \\
\text { ก }\end{array}$ & \%̊ & 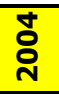 & ํㅗㅇ & 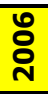 & 유 & $\begin{array}{l}\infty \\
\text { ㅇ } \\
\text { ㄱ }\end{array}$ & 용 & 웅 & 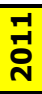 \\
\hline \multirow[t]{4}{*}{ BTEX } & benzene & & & & & 10 & & 2 & 27 & 8 & 13 & & 9 & & 3 & & 21 & & 1 & & & & \\
\hline & ethyl benzene & & & & & & & & & & & & 3 & 1 & & & 6 & & 1 & & & & \\
\hline & \begin{tabular}{|l|} 
toluene \\
\end{tabular} & & & & & & & & & & 9 & & 6 & & 3 & & 13 & & 1 & & & & \\
\hline & \begin{tabular}{|l}
$x y l e n e$ \\
\end{tabular} & & & & & & & & & & & & & & & & 6 & & 1 & & & & \\
\hline \multirow[t]{3}{*}{ DEP } & elemental carbon & & & & & & & 15 & 25 & 12 & 14 & 4 & 8 & 1 & 6 & 24 & 33 & 20 & 28 & & & & \\
\hline & organic carbon & & & & & & & 5 & & & 14 & 4 & 8 & 1 & 6 & 24 & 33 & 20 & 28 & & & & \\
\hline & total carbon & & & & & & & 11 & & & 14 & 4 & & 1 & 6 & 24 & 33 & 20 & 28 & & & & \\
\hline \multirow[t]{16}{*}{$\mathrm{PAH}$} & 1-nitropyrene & & & & & & & & & 8 & 10 & & 4 & & 2 & & 13 & & & & & & \\
\hline & acenaphthylene & & 16 & & & & & & & & & & & & & & 12 & & & & & & \\
\hline & anthracene & 3 & 46 & 1 & & 7 & & 10 & 25 & 12 & 13 & & 11 & & 3 & & 13 & & & & & & 1 \\
\hline & benzo(a)anthracene & & 8 & & & & & & & & & & & & & & 6 & & & & & & \\
\hline & benzo(a)pyrene & 3 & 48 & 1 & & 7 & & 10 & 25 & 12 & 13 & & 11 & & 3 & & 13 & & & & & & 1 \\
\hline & benzo(b)fluoranthene & & 8 & & & & & & & & & & & & & & 6 & & & & & & \\
\hline & benzo(e)pyrene & & 8 & & & & & & & & & & & & & & 6 & & & & & & \\
\hline & benzo $(g, h, i)$ perylene & & 8 & & & & & & & & & & & & & & 6 & & & & & & \\
\hline & benzo(k)fluoranthene & & 8 & & & & & & & & & & & & & & 6 & & & & & & \\
\hline & \begin{tabular}{|l|} 
chrysene \\
\end{tabular} & 3 & 48 & 1 & & 7 & & 10 & 25 & 12 & 13 & & 11 & & 3 & & 13 & & & & & & 1 \\
\hline & dibenzo(a,h)anthracene & & 8 & & & & & & & & & & & & & & 6 & & & & & & \\
\hline & fluoranthene & & 8 & & & & & & & & & & & & & & 6 & & & & & & \\
\hline & \begin{tabular}{|l|} 
fluorene \\
\end{tabular} & & 8 & & & & & & & & & & & & & & 6 & & & & & & \\
\hline & \begin{tabular}{|l|} 
indeno-1,2,3-cd-pyrene \\
\end{tabular} & & 8 & & & & & & & & & & & & & & 6 & & & & & & \\
\hline & phenanthrene & 3 & 48 & 1 & & 7 & & 10 & 25 & 12 & 13 & & 11 & & 3 & & 13 & & & & & & 1 \\
\hline & pyrene & 3 & 48 & 1 & & 7 & & 10 & 25 & 12 & 13 & & 11 & & 3 & & 13 & & & & & & 1 \\
\hline \multirow[t]{6}{*}{ aldehydes } & acetaldehyde & 8 & 41 & & & 6 & & 8 & 25 & 12 & 13 & & 8 & & 5 & & 11 & & & & & & \\
\hline & benzaldehyde & 8 & 41 & & & 6 & & 8 & 25 & 12 & 13 & & 8 & & 5 & & 11 & & & & & & \\
\hline & formaldehyde & 8 & 43 & & & 6 & & 8 & 25 & 12 & 13 & & 8 & 1 & 5 & & 11 & & & & & & \\
\hline & \begin{tabular}{|l|} 
glutaraldehyde \\
\end{tabular} & 8 & 41 & & & 6 & & 8 & 25 & 12 & 13 & & 8 & & 5 & & 11 & & & & & & \\
\hline & isovaleraldehyde & 8 & 41 & & & 6 & & 8 & 25 & 12 & 13 & & 8 & & 5 & & 11 & & & & & & \\
\hline & valeraldehyde & & & & & & & & 25 & 12 & 13 & & 8 & & 5 & & 11 & & & & & & \\
\hline \multirow[t]{2}{*}{ gases } & carbon monoxide & & 1 & & & & & & & & & & & & & 2 & 13 & 4 & 2 & & & & \\
\hline & nitrogen dioxide & 9 & 60 & 2 & 11 & 12 & 1 & 25 & 29 & 12 & 14 & & 15 & 12 & 4 & 3 & 19 & 3 & 3 & & & & \\
\hline
\end{tabular}


Table III: Descriptive statistics for PAHs (in $\mathrm{mg} / \mathrm{m}^{3}$ ), for all locomotive positions. (Parametric statistics could not be calculated due to the high percentage of non-detects.)

\begin{tabular}{|c|c|c|c|c|c|c|}
\hline \multirow[b]{2}{*}{ Substance } & \multirow[b]{2}{*}{ OEL } & \multirow[b]{2}{*}{$\mathbf{N}$} & \multirow[b]{2}{*}{$\%<M D C *$} & \multicolumn{3}{|c|}{ Order Statistics } \\
\hline & & & & Min & Max & Median \\
\hline 1-nitropyrene & - & 37 & 100.0 & $<0.0001$ & $<0.083$ & $<0.004$ \\
\hline acenaphthene & - & 14 & 100.0 & $<0.0002$ & $<0.001$ & $<0.001$ \\
\hline acenaphthylene & - & 14 & 100.0 & $<0.0002$ & $<0.001$ & $<0.001$ \\
\hline anthracene & - & 145 & 97.2 & $<0.0001$ & $<2.975$ & $<0.001$ \\
\hline benzo(a)anthracene & - & 14 & 100.0 & $<0.0005$ & $<0.007$ & $<0.002$ \\
\hline benzo(a)pyrene & - & 147 & 98.6 & $<0.0001$ & $<1.562$ & $<0.001$ \\
\hline benzo(b)fluoranthene & - & 14 & 100.0 & $<0.0002$ & $<0.001$ & $<0.001$ \\
\hline benzo(e)pyrene & - & 14 & 100.0 & $<0.0001$ & $<0.001$ & $<0.001$ \\
\hline benzo $(g, h, i)$ perylene & - & 14 & 100.0 & $<0.0003$ & $<0.001$ & $<0.001$ \\
\hline benzo(k)fluoranthene & - & 14 & 100.0 & $<0.0003$ & $<0.001$ & $<0.001$ \\
\hline chrysene & - & 147 & 95.9 & $<0.0001$ & $<3.125$ & $<0.001$ \\
\hline dibenzo(a,h)anthracene & - & 14 & 100.0 & $<0.0005$ & $<0.007$ & $<0.002$ \\
\hline fluoranthene & - & 14 & 100.0 & $<0.0004$ & $<0.002$ & $<0.001$ \\
\hline fluorene & - & 14 & 100.0 & $<0.0002$ & $<0.001$ & $<0.001$ \\
\hline indeno-1,2,3-cd-pyrene & - & 14 & 100.0 & $<0.0003$ & $<0.001$ & $<0.001$ \\
\hline phenanthrene & - & 147 & 98.0 & $<0.0001$ & $<3.125$ & $<0.001$ \\
\hline pyrene & - & 147 & 97.3 & $<0.0001$ & $<3.125$ & $<0.001$ \\
\hline
\end{tabular}

* MDC = minimum detectable concentration. 
Table IV: Descriptive statistics for Elemental Carbon $\left(\mathrm{OEL}=0.02 \mathrm{mg} / \mathrm{m}^{3}\right)$ in the lead locomotive.

\begin{tabular}{|c|c|c|c|c|c|c|c|c|c|}
\hline \multirow[t]{2}{*}{ Dataset } & \multirow[t]{2}{*}{$\mathbf{N}^{\mathbf{a}}$} & \multirow[t]{2}{*}{$\%<M Q C$} & \multicolumn{3}{|c|}{ Order Statistics } & \multicolumn{2}{|c|}{$\begin{array}{c}\text { Normal Distribution } \\
\text { Statistics }^{\text {b }}\end{array}$} & \multicolumn{2}{|c|}{$\begin{array}{c}\text { Lognormal } \\
\text { Distribution } \\
\text { Statistics }\end{array}$} \\
\hline & & & Min & Max & Median & Mean & SD & GM & GSD \\
\hline \multicolumn{10}{|c|}{ Lead Locomotive } \\
\hline $\begin{array}{l}\text { Manufacturer } \\
\text { EMD }\end{array}$ & 91 & $47.3 \%$ & $<0.0003$ & 0.0324 & $\begin{array}{c}0.0026 \\
(0.0022,0.0029)\end{array}$ & $\begin{array}{c}0.0026 \\
(0.0021,0.0036)\end{array}$ & - & 0.0017 & 2.55 \\
\hline GE & 65 & $72.3 \%$ & $<0.0013$ & 0.0212 & $\begin{array}{c}0.0028 \\
(0.0025,0.0031)\end{array}$ & $\begin{array}{c}0.0027 \\
(0.0014,0.0095)\end{array}$ & - & 0.0009 & 4.50 \\
\hline \begin{tabular}{|l} 
Class \\
4-axle
\end{tabular} & 55 & $43.6 \%$ & $<0.0003$ & 0.0148 & $\begin{array}{c}0.0028 \\
(0.0020,0.0031)\end{array}$ & $\begin{array}{c}0.0026 \\
(0.0019,0.0039)\end{array}$ & - & 0.0017 & 2.54 \\
\hline 6-axle & 101 & $65.4 \%$ & $<0.0011$ & 0.0324 & $\begin{array}{c}0.0027 \\
(0.0025,0.0030) \\
\end{array}$ & $\begin{array}{c}0.0029 \\
(0.0019,0.0058) \\
\end{array}$ & - & 0.0011 & 3.98 \\
\hline $\begin{array}{r}\text { Windows } \\
\text { Open }\end{array}$ & 58 & $55.2 \%$ & $<0.0003$ & 0.0324 & $\begin{array}{c}0.0027 \\
(0.0025,0.0031)\end{array}$ & $\begin{array}{c}0.0034 \\
0.0021,0.0078)\end{array}$ & - & 0.0015 & 3.74 \\
\hline Closed & 40 & $77.5 \%$ & $<0.0013$ & 0.0212 & $\begin{array}{c}0.0031 \\
(0.0028,0.0033)\end{array}$ & $\begin{array}{c}0.0022 \\
0.0009,0.0212) \\
\end{array}$ & - & 0.0008 & 4.26 \\
\hline
\end{tabular}

(a) Not all cases had information on the status of the windows during the run.

(b) Because all the EC subsets were censored, the mean was estimated using the Minimum Variance Unbiased Estimator (MVUE), which is calculated from the sample GM, sample GSD, and sample size (Hewett and Ganser, 2007). The confidence interval for the mean (i.e., MVUE) was calculated using a sample equal to the number of detects (rather than the full sample size. This results in a conservative (i.e., wider than probably necessary) confidence interval. (Hewett, 2006) 
Table V: Compliance statistics for Elemental Carbon $\left(\mathrm{OEL}=0.02 \mathrm{mg} / \mathrm{m}^{3}\right)$ in the lead locomotive.

\begin{tabular}{|c|c|c|c|c|c|}
\hline \multirow[b]{2}{*}{ Dataset } & \multirow[t]{2}{*}{$\mathbf{N}^{a}$} & \multicolumn{2}{|c|}{ Non-parametric ${ }^{b}$} & \multicolumn{2}{|c|}{ Lognormal Distribution $^{c}$} \\
\hline & & $\%>0 E L^{d}$ & $95^{\text {th }}$ Percentile ${ }^{d, e}$ & $\%>0 E L$ & $95^{\text {th }}$ Percentile \\
\hline \multicolumn{6}{|c|}{ Lead Locomotive } \\
\hline Manufacturer & & & & & \\
\hline EMD & 91 & $\begin{array}{c}2.2 \\
(0.4,6.8)\end{array}$ & $\begin{array}{c}0.0132 \\
(0.0053,0.0324)\end{array}$ & $\begin{array}{c}0.4 \\
(<0.1,1.7)\end{array}$ & $\begin{array}{c}0.0080 \\
(0.0059,0.0120)\end{array}$ \\
\hline GE & 65 & $\begin{array}{c}4.6 \\
(1.3,11.5) \\
\end{array}$ & $\begin{array}{c}0.0148 \\
(0.0074,0.0296) \\
\end{array}$ & $\begin{array}{c}3.2 \\
(0.6,11.6) \\
\end{array}$ & $\begin{array}{c}0.0136 \\
(0.0056,0.0582)\end{array}$ \\
\hline Class & & & & & \\
\hline 4-axle & 55 & $\begin{array}{c}0.0 \\
(0.0,5.3)\end{array}$ & $\begin{array}{c}0.0074 \\
(0.0044,-)\end{array}$ & $\begin{array}{c}0.4 \\
(<0.1,2.3)\end{array}$ & $\begin{array}{c}0.0079 \\
(0.0055,0.0133)\end{array}$ \\
\hline 6-axle & 101 & $\begin{array}{c}5.0 \\
(2.0,10.1) \\
\end{array}$ & $\begin{array}{c}0.0152 \\
(0.0076,0.0296)\end{array}$ & $\begin{array}{c}1.9 \\
(0.5,5.9) \\
\end{array}$ & $\begin{array}{c}0.0111 \\
(0.0067,0.0228)\end{array}$ \\
\hline Windows & & & & & \\
\hline Open & 58 & $\begin{array}{c}3.4 \\
(0.6,10.5)\end{array}$ & $\begin{array}{c}0.0152 \\
(0.0115,-)\end{array}$ & $\begin{array}{c}2.5 \\
(0.6,8.1)\end{array}$ & $\begin{array}{c}0.0131 \\
(0.0075,0.0300)\end{array}$ \\
\hline Closed & 40 & $\begin{array}{c}5.0 \\
(0.9,14.9) \\
\end{array}$ & $\begin{array}{c}0.0076 \\
(0.0050,-) \\
\end{array}$ & $\begin{array}{c}1.9 \\
(<0.1,14.9)\end{array}$ & $\begin{array}{c}0.0097 \\
(0.0033,0.0972)\end{array}$ \\
\hline
\end{tabular}

(a) Not all cases had information the status of the windows during the run

(b) - Includes, where appropriate, a non-parametric $90 \%$ confidence interval: $95 \% \mathrm{LCL}$ and $95 \% \mathrm{UCL}$ All of the EC subsets were censored. Therefore, the non-parametric confidence intervals are conservative (i.e., weighted toward the higher concentrations)

(c) Includes, where appropriate, a parametric $90 \%$ confidence interval calculated using the number of detects, rather than the full sample size: $95 \% \mathrm{LCL}$ and $95 \% \mathrm{UCL}$. This results in a conservative confidence interval; i.e., a confidence interval that is probably greatly than then $90 \%$ nominal width.

(d) The non-parametric exceedance fraction and $95^{\text {th }}$ percentile calculations include non-detects, even if greater than the OEL.

(e) The non-parametric $95 \% \mathrm{UCL}$ cannot be calculated if the sample size is less than 59. 
Table VI: AIHA exposure ratings, for Elemental Carbon $\left(\mathrm{OEL}=0.02 \mathrm{mg} / \mathrm{m}^{3}\right)$ in the lead locomotive, determined using Bayesian Decision Analysis (BDA). All BDA decision probabilities were calculated using a flat, non-informative prior. (The default parameter space was used unless otherwise indicated.)

\begin{tabular}{|c|c|c|c|c|c|c|c|c|c|}
\hline \multirow[b]{2}{*}{ Dataset } & \multirow[b]{2}{*}{$\mathbf{N}$} & \multirow{2}{*}{$\begin{array}{c}\text { Exposure } \\
\text { Rating }\end{array}$} & \multirow{2}{*}{$\begin{array}{l}\text { Certainty } \\
\text { Level }\end{array}$} & \multicolumn{5}{|c|}{$\begin{array}{c}\text { Bayesian Decision Analysis } \\
\text { Decision Probabilities Per Exposure Category * }\end{array}$} & \multirow[t]{2}{*}{ Notes } \\
\hline & & & & $\mathbf{0}$ & 1 & 2 & 3 & 4 & \\
\hline \multicolumn{10}{|c|}{ Lead Locomotive } \\
\hline Manufacturer $=\mathrm{EMD}$ & 91 & 2 & high & 0.000 & 0.000 & 0.852 & 0.148 & 0.000 & \\
\hline Manufacturer = GE & 65 & 3 & medium & 0.000 & 0.000 & 0.240 & 0.697 & 0.099 & 1 \\
\hline Class $=4$-axle & 55 & 2 & high & 0.000 & 0.000 & 0.791 & 0.210 & 0.000 & \\
\hline Class $=6$-axle & 101 & 3 & medium & 0.000 & 0.000 & 0.257 & 0.712 & 0.032 & 1 \\
\hline Windows = Open & 58 & 3 & medium & 0.000 & 0.000 & 0.109 & 0.734 & 0.153 & 1 \\
\hline Windows = Closed & 40 & 2 or 3 & high & 0.000 & 0.000 & 0.568 & 0.393 & 0.039 & 1 \\
\hline
\end{tabular}

* Exposure categories $0,1,2$, and 3 refer to exposure profiles that have $95^{\text {th }}$ percentiles that are less than $1 \%, 10 \%, 50 \%$, and $100 \%$ of the exposure limit, respectively. A category 4 exposure profile has a $95^{\text {th }}$ percentile that exceeds the exposure limit.

1 - The sample GSD (calculated using MLE) approached or exceeded the default value of 4 for the maximum GSD. The maximum GSD for parameter space was increased to 8 . 
Table VII: Comparison of the mean Elemental Carbon concentrations for CSXT lead locomotives to values reported in the literature for other industries, urban settings, and rural settings.

\begin{tabular}{|c|c|c|c|c|c|}
\hline Industry & Area/Mine/Job & Year & $\mathbf{N}$ & Mean & Reference \\
\hline \multirow{14}{*}{ Mining } & limestone & \multirow{14}{*}{$1998-2000$} & 108 & 0.384 & \multirow{14}{*}{ Coble et al. (2010) } \\
\hline & potash & & 124 & 0.191 & \\
\hline & & & 84 & 0.094 & \\
\hline & salt & & 118 & 0.082 & \\
\hline & \multirow{3}{*}{ trona } & & 126 & 0.040 & \\
\hline & & & 116 & 0.084 & \\
\hline & & & 103 & 0.071 & \\
\hline & \multirow{7}{*}{ surface } & & 33 & 0.006 & \\
\hline & & & 61 & 0.004 & \\
\hline & & & 35 & 0.003 & \\
\hline & & & 25 & 0.004 & \\
\hline & & & 31 & 0.002 & \\
\hline & & & 33 & 0.002 & \\
\hline & & & 47 & 0.003 & \\
\hline \multirow{4}{*}{ Trucking } & local & \multirow[t]{2}{*}{$2001-2005$} & 576 & 0.0016 & \multirow[t]{2}{*}{ Davis et al. (2007) } \\
\hline & long haul & & 349 & 0.0014 & \\
\hline & local & \multirow{4}{*}{ 1988-1989 } & 56 & 0.0054 & \multirow{4}{*}{ Zaebst et al. (1991) } \\
\hline & long haul & & 72 & 0.0051 & \\
\hline \multirow{16}{*}{ Background } & highway & & 21 & 0.0034 & \\
\hline & residential & & 23 & 0.0014 & \\
\hline & highway & \multirow[t]{2}{*}{1999} & 21 & 0.0043 & \multirow[t]{2}{*}{ Lena et al. (2002) } \\
\hline & residential & & 9 & 0.0026 & \\
\hline & rural & $1999-2000$ & na & 0.001 & Chen et al. (2001) \\
\hline & \multirow{9}{*}{ urban } & 1996 & 20 & 0.0034 & Kinney et al. (2000) \\
\hline & & 1999 & 27 & 0.0027 & Liukonen et al. (2002)* \\
\hline & & $2000-2001$ & 43 & 0.0035 & O'Kelly (2001) \\
\hline & & $2001-2002$ & na & 0.0021 & $\mathrm{Na}$ et al. (2004) \\
\hline & & \multirow{4}{*}{1999} & na & 0.0033 & \multirow{4}{*}{ Tolocka et al. (2001) } \\
\hline & & & na & 0.0033 & \\
\hline & & & na & 0.0025 & \\
\hline & & & na & 0.0015 & \\
\hline & & 1995 & na & 0.0038 & $\begin{array}{l}\text { Table } 1 \text { of } \mathrm{Na} \text { et al. (2004) } \\
\text { (source: Kim et al., 2000) }\end{array}$ \\
\hline & average population outdoor & 1995 & na & 0.0022 & \\
\hline & average population indoor & & na & 0.0015 & CA-EPA (1998) \\
\hline & trailing & 1997 & 7 & 0.017 & Liukonen et al. (2002) \\
\hline & lead & $1999-2000$ & 71 & 0.0015 & Seshagiri $(2001,2003)$ \\
\hline Railroad & trailing & & 69 & 0.0078 & \\
\hline & workers & 1996 & 18 & 0.0084 & Verma et al. (1999) \\
\hline & lead & $1996-2002$ & 31 & 0.0031 & Finkelstein and Verma (2002) \\
\hline & trailing & & 24 & 0.012 & \\
\hline & CSXT trailing & $1990-2007$ & 22 & 0.0111 & (this report) \\
\hline & CSXT lead & & 156 & 0.0028 & \\
\hline
\end{tabular}


Table VIII: Analysis of Variance (ANOVA) results for evaluating the effect of locomotive position (lead vs trailing) and window status (open vs closed).

\begin{tabular}{|c|c|c|c|c|c|}
\hline Source & SS & df & Mean Squares & F-ratio & p-value \\
\hline Position & 3.27815 & 1 & 3.27815 & 16.67934 & 0.00008 \\
\hline Windows & 0.26342 & 1 & 0.26342 & 1.34031 & 0.24940 \\
\hline Position*Windows & 0.03761 & 1 & 0.03761 & 0.19135 & 0.66262 \\
\hline Error & 22.40552 & 114 & 0.19654 & & \\
\hline
\end{tabular}

Table IX: Comparison of the mean Elemental Carbon concentrations for CSXT lead locomotives to values reported in the literature for locomotives.

\begin{tabular}{|c|c|c|c|c|c|}
\hline Scenario & $\mathbf{N}$ & $\begin{array}{l}\text { Number } \\
\text { of Studies }\end{array}$ & $\begin{array}{c}\text { Mean } \\
\left(\text { in } \mathrm{mg} / \mathrm{m}^{3}\right)\end{array}$ & Reference & Notes \\
\hline \multicolumn{5}{|c|}{ CSXT } & \\
\hline $\begin{array}{l}\text { CSXT Locomotive crew } \\
\text { Lead locomotive } \\
\text { Trailing locomotive } \\
\text { Other locomotive }\end{array}$ & $\begin{array}{c}190 \\
156 \\
22 \\
11\end{array}$ & 1 & $\begin{array}{l}0.0037 \\
0.0028 \\
0.0111 \\
0.0032\end{array}$ & (this report) & \\
\hline \multicolumn{5}{|c|}{ Other Studies } & \\
\hline Trailing locomotive & 7 & 1 & 0.017 & Liukonen et al. (2002) & $a$ \\
\hline $\begin{array}{l}\text { Lead locomotive } \\
\text { Trailing locomotive }\end{array}$ & $\begin{array}{l}47 \\
47\end{array}$ & 1 & $\begin{array}{l}0.0020 \\
0.0101\end{array}$ & Seshagiri $(2001,2003)$ & $b$ \\
\hline $\begin{array}{l}\text { Locomotive crew } \\
\text { Maintenance }\end{array}$ & & $\begin{array}{l}4 \\
2\end{array}$ & $\begin{array}{l}0.006 \text { to } 0.020 \\
0.005 \text { to } 0.039\end{array}$ & $\begin{array}{l}\text { Pronk, Coble, and } \\
\text { Stewart (2009) }\end{array}$ & \\
\hline $\begin{array}{l}\text { Rail yard } \\
\text { Workers - Respirable EC } \\
\text { Yard locomotive EC }\end{array}$ & $\begin{array}{c}18 \\
9\end{array}$ & 1 & $\begin{array}{l}0.0084 \\
0.0178\end{array}$ & Verma et al. (1999) & C \\
\hline $\begin{array}{l}\text { Rail yard - foreman and } \\
\text { switchman } \\
\text { Total EC } \\
\text { Respirable EC }\end{array}$ & $\begin{array}{c}10 \\
5\end{array}$ & 1 & $\begin{array}{l}0.0065 \\
0.0023\end{array}$ & NIOSH (2011) & $\begin{array}{l}c \\
d\end{array}$ \\
\hline
\end{tabular}

a - Six of the seven measurements were from the trailing locomotive or "long hood forward" (where the cab was behind the engine exhaust stacks) (Since the Railroad A measurements were provided by CSXT only the seven Railroad B measurements were used.) b - Calculated from the original data listed in Seshagiri (2001).

c - No size selective device was used. This is identical to the method used by CSXT.

d - A size-selective device (i.e., cyclone) was used to prevent the larger, non-diesel exhaust organic particles from being collected. 


\section{References}

ACGIH (American Conference of Governmental Industrial Hygienists) (2012): 2012 TLVs and BEIs - Threshold Limit Values for Chemical Substances and Physical Agents and Biological Exposure Indices. ACGIH, Cincinnati, Ohio.

AIHA (American Industrial Hygiene Association) (2011): Emergency Response Planning Guidelines and Workplace Environmental Exposure Levels Handbook. American Industrial Hygiene Association, Fairfax, VA.

AIOH (Australian Institute of Occupational Hygienists) (2013): Diesel Particulate Matter and Occupational Health Issues. (www.aioh.org.au).

Cal-EPA (1998): The Report on Diesel Exhaust. California Environmental Protection Agency, Air Resources Board.

CDHS (California Department of Health Services) (2002): Health Hazard Advisory - Diesel Engine Exhaust. Hazard Evaluation System \& Information Service (November).

Chen, L.W.A., Doddridge, B.G., Dickerson, R.R., Chow, J.C., Mueller, P.K., Quinn, J., and Butler, W.A. (2001): Seasonal variations in elemental carbon aerosol, carbon monoxide and sulfur dioxide: implication for sources. Geophysical Research Letters 28(9):1711-1714.

Coble, J.B., Stewart, P.A., Vermeulen, R., et al. (2010): The Diesel Exhaust in Miners Study: II. Exposure Monitoring Surveys and Development of Exposure Groups. Annals of Occupational Hygiene 54:747-761.

Davis, M.E., Smith, T.J., Laden, F., et al . (2007): Driver Exposure to Combustion Particles in the U.S. Trucking Industry. Journal of Occupational and Environmental Health 4:848-854.

EPA (Environmental Protection Agency) (2002): Health Assessment Document for Diesel Engine Exhaust. U.S. Environmental Protection Agency, Washington, DC.

Finkelstein, M.M. and Verma, D.K. (2002): Cancer Risk from Diesel Exhaust Exposure in the Canadian Railroad Industry: A Feasibility Study. in "Special Report - Research Directions to Improve Estimates of Human Exposure and Risk from Diesel Exhaust", Health Effects Institute, April 2002.

Kim, B.M., Teffera, S., and Zeldin, M.D. (2000): Characterization of $\mathrm{PM}_{2.5}$ and $\mathrm{PM}_{10}$ in the south coast air basin of Southern California: Part 1 - spatial variations. Journal of the Air and Waste Management Association 50:2034-2044.

Kinney, K.L., Aggarwal, M., Northridge, M.E., Janssen, N.A.H., and Shepard, P. (2000): Airborne Concentrations of $\mathrm{PM}_{2.5}$ and Diesel Exhaust Particles on Harlem Sidewalks: A Community-based Pilot Study. Environmental Health Perspectives 108:213-218.

Liukonen, L.R., Grogan, J.L., and Myers, W. (2002): Diesel Particulate Matter Exposure to Railroad Train Crews. American Industrial Hygiene Association Journal 63:610-616.

Lena, T.S., Ochieng, V., Carter, M., Holguin-Veras, J., and Kinney, P.L. (2002): Elemental Carbon and PM 2.5 Levels in a Urban Community Heavily Impacted by Truck Traffic. Environmental Health Perspectives 110:1000-1015.

MSHA (Mine Safety and Health Administration) (2013): Title 30 Code of Federal Regulations Part 57.5060(b) Diesel Particulate Matter-Underground Only.

$\mathrm{Na}$, K., Sawant, A.A., Song, C., and Cocker, D.R. (2004): Primary and secondary carbonaceous species in the atmosphere of Wester Riverside County, California. Atmospheric Environment 38:1345-1355.

NIOSH (National Institute for Occupational Safety and Health) (2004): NIOSH Pocket Guide to Chemical Hazards. Department of Health and Human Services, Centers for Disease Control, NIOSH. Publication No. 97-140.

NIOSH (National Institute for Occupational Safety and Health) (2011): Health Hazard Evaluation Report HETA 20110045-3149 - Evaluation of Prostate Cancer, Diesel Exhaust Exposures, and Radio Frequency Exposures Among Employees at a Rail Yard - Alabama. Department of Health and Human Services, Centers for Disease Control, NIOSH.

O'Kelly, J.C. (2001): Mira Loma PM 10 Monitoring. South Coast Air Quality Management District Monitoring and Analysis, Report Number MA-2001-08.

OSHA (Occupational Safety and Health Administration) (2012): Code of Federal Regulations 29, Part 1910, Subpart Z. 
Pronk, A., Coble, J., and Steward, P.A. (2009): Occupational exposure to diesel engine exhaust: A literature review. Journal of Exposure Science and Environmental Epidemiology 19:443-457.

Seshagiri, B. (2001): Exposure to Diesel Exhaust Emissions and Noise on Board Locomotives. Human Resources and Development Canada, Ottawa, Canada.

Seshagiri, B. (2003): Exposure to Diesel Exhaust Emissions on Board Locomotives. American Industrial Hygiene Association Journal 64:678-683.

Tolocka, M.P., Solomon, P.A., Mitchell, W., et al. (2001): East versus West in the US: Chemical Characteristics of $\mathrm{PM}_{2.5}$ during the Winter of 1999. Aerosol Science and Technology 34:88-96.

Verma, D.K., Shaw, L., Julian, J. et al . (1999): A Comparison of Sampling and Analytical Methods for Assessment Occupational Exposure to Diesel Exhaust in a Railroad Work Environment. Applied Occupational and Environmental Hygiene 14:701-714.

Zaebst, D.D., Clapp, D.E., Blade, L.M., et al . (1991): Quantitative Determination of Trucking Industry Workers' Exposures to Diesel Exhaust Particles. American Industrial Hygiene Association Journal 52:529-541. 